\title{
Resveratrol alleviates ischemia/reperfusion injury of diabetic myocardium via inducing autophagy
}

\author{
XIANFENG QU ${ }^{1}$, XIAO CHEN $^{1}$, QINGQING SHI ${ }^{1}$, XIAOFEI WANG ${ }^{1}$, DONGGUO WANG $^{2}$ and LI YANG $^{1}$ \\ ${ }^{1}$ Department of Anesthesiology, Municipal Hospital of Taizhou; ${ }^{2}$ Clinical Laboratory, \\ Municipal Hospital of Taizhou, Taizhou, Zhejiang 318000, P.R. China
}

Received October 21, 2018; Accepted June 13, 2019

DOI: $10.3892 /$ etm.2019.7846

\begin{abstract}
The rising incidence and complications of diabetes constitutes a major public health issue. The mortality rate of diabetes-induced myocardial ischemia/reperfusion (I/R) injury is significantly elevated. Resveratrol (RSV) is a naturally occurring polyphenol considered to be a potent cardioprotective compound. The aim of the present study was to explore the function and molecular mechanism of RSV on diabetes-induced myocardial I/R injury. Left anterior descending coronary artery ligation was performed to stimulate myocardial I/R injury in streptozotocin-induced diabetic rats. Heart electrical activity was monitored through an electrocardiogram to confirm successful models. The myocardial infarct volume was detected via 2,3,5-triphenyltetrazolium chloride staining. Western blotting was employed to examine the levels of autophagy markers. It was found that the injection of RSV mitigated the ischemia- or I/R injury-induced myocardial damage on hemodynamic function and infarct size, but the autophagy inhibitor 3-methyladenine significantly blocked the function of RSV. Furthermore, the application of RSV significantly enhanced the expression of Beclin-1 and LC-3II but inhibited the serum levels of tumor necrosis factor- $\alpha$ and interleukin-6. These findings revealed an alleviating effect of RSV on diabetes-induced myocardial I/R injury and provided new evidence for the successful application of RSV on the diabetic myocardium.
\end{abstract}

\section{Introduction}

Economic growth and improvement of living standards has led to an increase in the prevalence of diabetes (1). Coronary artery disease causing myocardial infarction is one of the main complications associated with diabetes (2). The mortality rate

Correspondence to: Dr Li Yang, Department of Anesthesiology, Municipal Hospital of Taizhou, 381 East Zhong-Shan Road, Jiao-Jiang, Taizhou, Zhejiang 318000, P.R. China

E-mail: doctoreinstein@126.com

Key words: resveratrol autophagy, diabetes, ischemia/reperfusion injury of diabetic patients following myocardial infarction is significantly increased, when compared with nondiabetic patients (3). Previous studies showed some key mechanisms involved in the sensitivity of the diabetic heart to myocardial infarction, such as extracellular signal regulated kinase (ERK)1/2 signaling (4), inflammatory (5) and phosphatidylinositol 3 kinase $(\mathrm{PI} 3 \mathrm{~K}) /$ protein kinase B (AKT)/glycogen synthase kinase (GSK)-3 $\beta$ (6). Hyperglycemia is the major characteristic of diabetes, which causes reactive oxygen species overproduction and exacerbates myocardial infarction (7). Therefore, excessive autophagy is a major mechanism of myocardial ischemia reperfusion injury in diabetes (8). In ischemic patients, reperfusion to the ischemic heart mitigates the mortality rate (9); however, reperfusion also causes further myocardial dysfunction (10). It is therefore urgent to explore effective drugs against diabetic myocardial ischemia/reperfusion (I/R) injury.

Resveratrol (RVS), otherwise known as 3,4',5-trihydroxytransstilbene, a polyphenolic compound present in grapes, wines, peanuts and several other fruits and vegetables, has been associated with increased longevity and shown to have a positive effect in the treatment of cancer and cardiovascular disease (11). The targets of RSV include various pathways and molecules, such as sirtuins, FOXOs, and autophagy and inflammatory cytokines (12). RSV has been shown to protect against cerebral I/R injury by inhibiting NACHT, LRR and PYD domains-containing protein 3 (NLRP3) inflammasome activation, revealing the relationship between RSV and inflammatory response (13). Autophagy is a self-clearing process that removes dying or unwanted cells and is accompanied by the activation of autophagy markers Beclin 1 and light chain 3-II (LC3-II) (14). It has been reported that autophagy significantly contributes to the degree of myocardial I/R injury (15), suggesting that therapeutic manipulation of autophagy in the myocardium may benefit post-infarction cardiac healing and remodeling. Previous studies have shown that RSV reduces the severity of myocardial $\mathrm{I} / \mathrm{R}$ injury by inducing autophagy $(16,17)$. Therefore, RSV has been proposed as an effective therapy for the prevention of myocardial injury (18). It has also been reported that RSV alleviates myocardial dysfunction in diabetes and can serve as a potent pharmacological agent in reducing diabetic-induced I/R injury (3). However, its molecular mechanism remains unclear.

In the present study, the diabetic rat model was constructed through a high-fat diet combined with a streptozotocin 
(STZ) injection. Diabetic rats were subjected to $30 \mathrm{~min}$ of ischemia and $2 \mathrm{~h}$ of reperfusion to stimulate diabetic-induced myocardial I/R injury. It was found that the injection of RSV mitigated the ischemia- or I/R injury-induced myocardial damage on hemodynamic function and infarct size, but the autophagy inhibitor 3-methyladenine (3-MA) significantly blocked the function of RSV. Furthermore, the application of RSV significantly enhanced the expression of Beclin-1 and LC-3II but blocked the serum levels of cytokines tumor necrosis factor- $\alpha$ (TNF)- $\alpha$ and interleukin (IL)- 6 . In addition, the application of 3-MA suppressed the function of RSV. In conclusion, RSV alleviates I/R injury of the diabetic myocardium via inducing the expression of autophagy markers Beclin-1 and LC-3II and inhibiting expression of TNF- $\alpha$ and IL-6. These findings revealed an alleviating effect of RSV on diabetic-induced myocardial I/R injury, as well as its molecular basis, providing a novel strategy for diabetic myocardial I/R injury therapy.

\section{Materials and methods}

Animals and grouping. A total of 100 male Sprague-Dawley rats [Certificate no. SCXK(Zhe) 2013-0003] aged 6-8 weeks and weighing $200 \pm 20 \mathrm{~g}$ were purchased from the Experimental Animal Center of Zhejiang Province (Taizhou, Zhejiang, China). Rats were housed at $24 \pm 1^{\circ} \mathrm{C}$ with $40-50 \%$ humidity in a clean environment kept to a 12 -h light/dark cycle. All animals had unlimited access to food and water. All experimental procedures were approved by the Experimental Animal Ethics Committee of Municipal Hospital of Taizhou. Rats were given a high-fat diet (20\% sugar, $10 \%$ lard, $10 \%$ egg yolk powder, $2 \%$ cholesterol and 58\% common chow). After 2 months, rats were intraperitoneally injected with STZ $(30 \mathrm{mg} / \mathrm{kg}$; Enzo Life Sciences, Inc.) dissolved in $0.1 \mathrm{mM}$ citrate buffer (pH 4.5) according to a previous study (19). The fasting blood glucose (based on tail vein samples) was detected once a week for 4 weeks. Rats with a fasting blood glucose of $\geq 16.7 \mathrm{mM}$ were selected as diabetic animals for follow-up experiments. Diabetic rats were randomly divided into four groups $(n=20$ per group), the Control, Model, RSV (Merck KGaA) and 3-MA groups. Diabetic rats in the RSV group were intragastrically administered RSV (10 mg/kg/d). Rats in the 3-MA group were intravenously administered 3-MA (15 mg/kg/d), based on the RSV injection. The Control and Model groups were injected with the same amount of physiological saline.

Animal model of myocardial I/R. Rats were anesthetized with $10 \%$ chloral hydrate $(300 \mathrm{mg} / \mathrm{kg}$ ) and exhibited no signs of peritonitis. A thoracotomy was performed exposing the heart. The left anterior descending (LAD) coronary artery was ligated to stimulate myocardial infarction for $30 \mathrm{~min}$. Electrocardiogram (ECG) ST Segment elevation signified a successful infarction. After $30 \mathrm{~min}$, the ligation was removed to restore blood flow for an additional $2 \mathrm{~h}$, to stimulate reperfusion. Rats in the Model, RSV and 3-MA groups were treated according to the above procedure, and the control group underwent a sham surgery.

Measurement of hemodynamic function of the heart. Hemodynamic function was assessed prior to modeling (baseline), during trachea cannula (before ischemia) and during ischemia and I/R. The left ventricular systolic pressure (LVSP) and time derivatives of the pressure change were measured during contraction $(+\mathrm{dP} / \mathrm{dt})$ and relaxation $(-\mathrm{dP} / \mathrm{dt})$ using a polygraph, as previously described (16). A MedLab bio-signal system (Nanjing Medease Science and Technology Co., Ltd.) was used to monitor electrical activity in the heart through an ECG throughout the experiment.

Measurement of myocardial infarct size. A total of six rats in each group were randomly selected for the measurement of myocardial infarct size. The left ventricle was rapidly excised from the anesthetized rat, weighed and cut into 1-2 mm thick sections. Viable tissues were stained in red with 2\% 2,3,5-triphenyltetrazolium chloride (TTC) for $20 \mathrm{~min}$ at $37^{\circ} \mathrm{C}$. Images were captured of the stained sections and the pale-appearing infarct area was calculated using Image Pro Plus 4.5 software (Media Cybernetics, Inc.). The infarct ratio (\%) was calculated by dividing the infarct volume by the total volume of the sections.

Determination of the content of TNF- $a$ and $I L-6$. A total of $200 \mu \mathrm{l}$ of blood were collected from the tails veins. After centrifugation at $4,000 \times \mathrm{g}$ for $8 \mathrm{~min}$ at $4^{\circ} \mathrm{C}$, sera were collected for follow-up experiments. Serum levels of TNF- $\alpha$ (cat. no. JM-01587R1) and IL-6 (cat. no. JM-01597R1) were assessed with enzyme-linked immunosorbent assay (ELISA) kits (Jingmei Biological Technology, Co., Ltd.).

Western blotting. Isolated rat cardiomyocytes were homogenized into pieces for protein extraction. Proteins were extracted using RIPA buffer (Sigma-Aldrich; Merck KGa) supplemented with $1 \mathrm{mM}$ PMSF (Sigma-Aldrich; Merck KGaA). The concentration was determined with bicinchoninic acid assay kit (Pierce; Thermo Fisher Scientific, Inc.). The same amount of protein $(10 \mu \mathrm{g} /$ lane) was separated by $12 \%$ SDS-PAGE using a constant voltage of $80 \mathrm{~V}$ for $120 \mathrm{~min}$. The proteins were transferred into polyvinylidene fluoride membrane (EMD Millipore). The membrane was blocked with 5\% non-fat milk in Tris-buffered saline (TBS) for $1 \mathrm{~h}$ at room temperature. After the blockage, the membrane was incubated with the primary antibodies (all Abcam), including rabbit monoclonal anti-PI3K $\gamma$ (ab32089; 1:1,000), anti-Beclin 1 (ab207612; 1:2,000), anti-LC-3B (ab192890; 1:2,000) and anti-GAPDH (ab181602; 1:10,000) antibodies overnight at $4^{\circ} \mathrm{C}$. The next morning, the membrane was washed with TBS containing $0.05 \%$ Tween-20 (TBST) and probed with horseradish peroxidase-conjugated goat anti-rabbit IgG H\&L (ab6721; $1: 10,000$; Abacm) at $37^{\circ} \mathrm{C}$ for $1 \mathrm{~h}$. After being washed with TBST, the membranes were developed using Enhanced chemiluminescence kit (PerkinElmer, Inc.) and visualized using an Odyssey Infrared Imaging System (LI-COR Biosciences). The bands were quantified using ImageJ software (v1.51; National Institutes of Health) by measuring the target gene band intensity normalized to the internal control GAPDH.

Statistical analysis. All data are presented as the mean \pm standard deviation for three independent experiments and analyzed by SPSS software v16.0 (SPSS, Inc.). The statistical significance among the four experimental groups was 

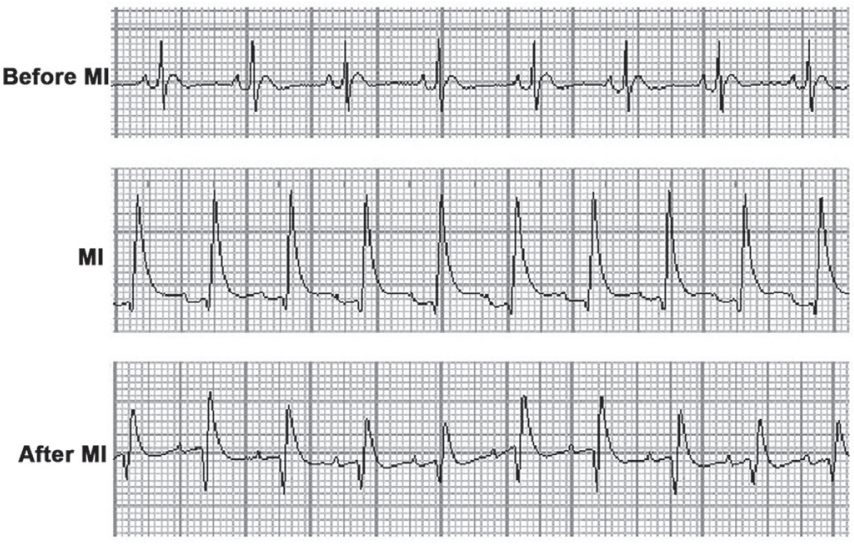

Figure 1. Heart electrical activity is detected using ECG. Left anterior descending artery ligation/reperfusion surgery was performed on rats from different groups to construct the myocardial I/R injury model. The traces of rats' ECG were examined prior to and during $\mathrm{MI}$ and following I/R injury. $\mathrm{I} / \mathrm{R}$, ischemia/reperfusion; ECG, electrocardiogram; MI, myocardial ischemia.

determined by a multivariate analysis of variance followed by Tukey post hoc test. $\mathrm{P}<0.05$ was considered to indicate a statistically significant difference.

\section{Results}

The myocardial I/R rat model is successfully constructed. In order to examine the myocardial I/R rat model, a MedLab bio-signal system was used to monitor the heart's electrical activity through an ECG throughout the experiment (Fig. 1). Prior to myocardial ischemia, ECG results appeared normal with a normal ST segment. Following LAD coronary artery ligation for $30 \mathrm{~min}$, A significant ST elevation was observed, suggesting successful ischemia. Following blood flow recovery for $2 \mathrm{~h}$, the ST elevation was reduced to $\sim 30-40 \%$ of its baseline values, indicating successful I/R injury. These data indicated the successful construction of the myocardial I/R rat model for follow-up experiments.

$R S V$ mitigates ischemia and I/R injury on heart function. To investigate the effect of RSV on myocardial function following $\mathrm{I} / \mathrm{R}$ injury, hemodynamic parameters were measured in all groups throughout the experiments (Fig. 2). No differences were identified in the baseline, LVSP, $+\mathrm{dP} / \mathrm{dt}$ and $-\mathrm{dP} / \mathrm{dt}$ values among the four groups $(\mathrm{P}>0.05$, Fig. $2 \mathrm{~A}-\mathrm{C})$. Prior to myocardial ischemia, no difference was observed in the LVSP, $+\mathrm{dP} / \mathrm{dt}$ and $-\mathrm{dP} / \mathrm{dt}$ values between the Control and model groups ( $\mathrm{P}>0.05$, Fig. 2A-C). However, when compared with the Model group, pretreatment with RSV significantly upregulated LVSP, $+\mathrm{dP} / \mathrm{dt}$ and $-\mathrm{dP} / \mathrm{dt}(\mathrm{P}<0.05$, Fig. 2A-C). During ischemia, LVSP, $+\mathrm{dP} / \mathrm{dt}$ and $-\mathrm{dP} / \mathrm{dt}$ in the Model group were significantly downregulated, as compared with the control group ( $\mathrm{P}<0.05$, Fig. 2A-C). The addition of RSV mitigated the blockage induced by ischemia, whereas the application of 3-MA suppressed the function of RSV on LVSP, $+\mathrm{dP} / \mathrm{dt}$ and -dP/dt (Fig. 2A-C). Similar trends were observed during I/R damage. These data suggest that RSV pretreatment mitigated the ischemia and I/R injury on heart function, but 3-MA suppressed that function of RSV.

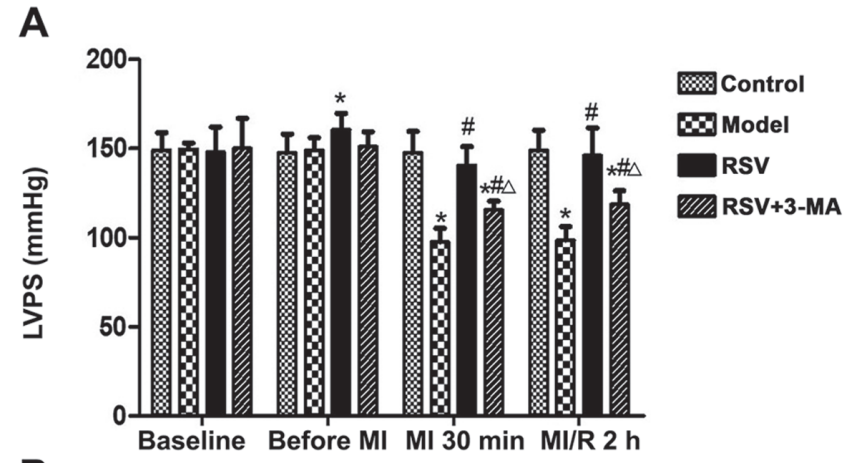

B
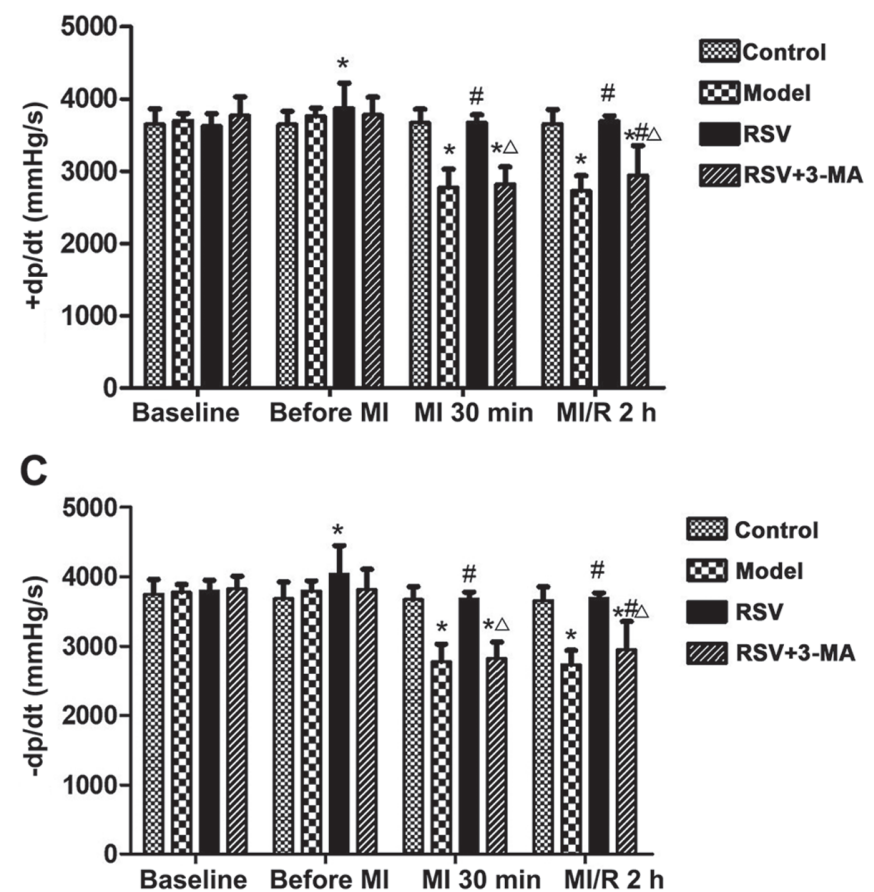

Figure 2. RSV and 3-MA affects the myocardial function via heart hemodynamic function examination. RSV and 3-MA modulated the levels of (A) LVSP, (B) $+\mathrm{dP} / \mathrm{dt}$ and $(\mathrm{C})-\mathrm{dP} / \mathrm{dt}$. Diabetic rats in the RSV group were given RSV $(10 \mathrm{mg} / \mathrm{kg} / \mathrm{d})$ by intragastric administration. Rats in the 3-MA (RSV+3-MA) group were given an intravenous injection of 3-MA $(0.015 \mathrm{~g} / \mathrm{kg} / \mathrm{d})$ based on the RSV $(10 \mathrm{mg} / \mathrm{kg} / \mathrm{d})$ injection. The Control and Model groups were injected with the same amount of physiological saline. After 7 days, LAD coronary artery was performed on rats in the Model, RSV and RSV+3-MA groups to stimulate myocardial infarction. The control group underwent a sham surgery without LAD coronary artery ligation. The hemodynamic parameters were measured prior to modeling (baseline), during trachea cannula (before ischemia) and during ischemia and $\mathrm{I} / \mathrm{R}$. ${ }^{*} \mathrm{P}<0.05$ vs. the control group; ${ }^{\#} \mathrm{P}<0.05$ vs. the Model group; ${ }^{\wedge} \mathrm{P}<0.05$ vs. the RSV group. RSV, resveratrol; 3-MA, 3-methyladenine; LVSP, left ventricular systolic pressure; - $\mathrm{dP} / \mathrm{dt}$, time derivatives of the pressure change during contraction; $-\mathrm{dP} / \mathrm{dt}$, time derivatives of the pressure change during relaxation; $\mathrm{LAD}$, left anterior descending; I/R, ischemia/reperfusion; MI, myocardial ischaemia.

RSV mitigates the myocardial infarct size caused by ischemia and $I / R$. The myocardial infarct size was measured following different treatments. Fig. 3A shows the typical morphological characteristics of myocardial sections following TTC staining. An extensive pale infarct area was observed in the myocardial sections of rats in the Model group, which was not there in the control group. Pretreatment with RSV significantly decreased the infarct area $(\mathrm{P}<0.05)$. However, the pale infarct area in the myocardial sections of the 3-MA group was significantly 

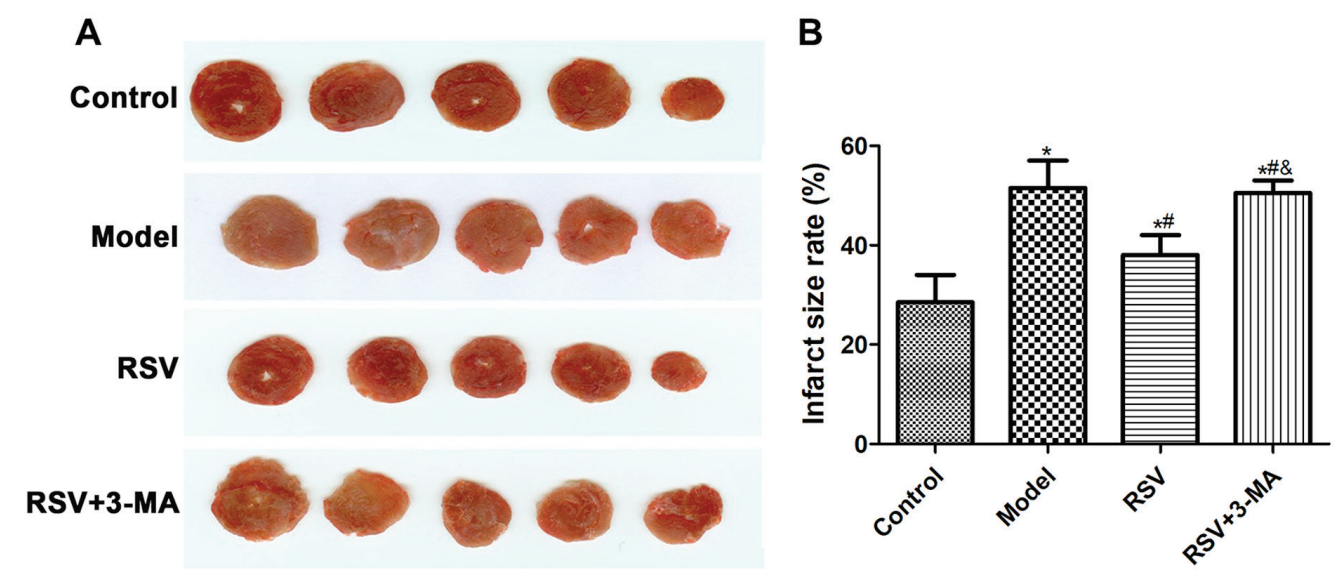

Figure 3. RSV and 3-MA affects the infarct size. (A) Visualization of the typical morphological characteristics of myocardial sections by TTC staining. Diabetic rats in the RSV group were given RSV $(10 \mathrm{mg} / \mathrm{kg} / \mathrm{d})$. Rats in the 3-MA (RSV+3-MA) group were administered 3-MA (0.015 g/kg/d), based on the RSV $(10 \mathrm{mg} / \mathrm{kg} / \mathrm{d})$ injection. The Control and Model groups were injected with the same amount of physiological saline. After 7 days, LAD coronary artery was performed on rats in the Model, RSV and RSV+3-MA groups to stimulate myocardial I/R injury. The control group underwent a sham surgery. The left ventricles from 6 rats in each group were extracted and cut into 1-2-mm thick sections for TTC staining. Red represents normal tissue and pale stands for infarct tissue. (B) The infarct ratio (\%) was calculated by dividing the infarct volume by the total volume of the sections. " $\mathrm{P}<0.05$ vs. the control group; ${ }^{\# P}<0.05$ vs. the Model group; ' $\mathrm{P}<0.05$ vs. the RSV group. RSV, resveratrol; 3-MA, 3-methyladenine; TTC, 2,3,5-triphenyltetrazolium chloride; LAD, left anterior descending; $\mathrm{I} / \mathrm{R}$, ischemia/reperfusion.
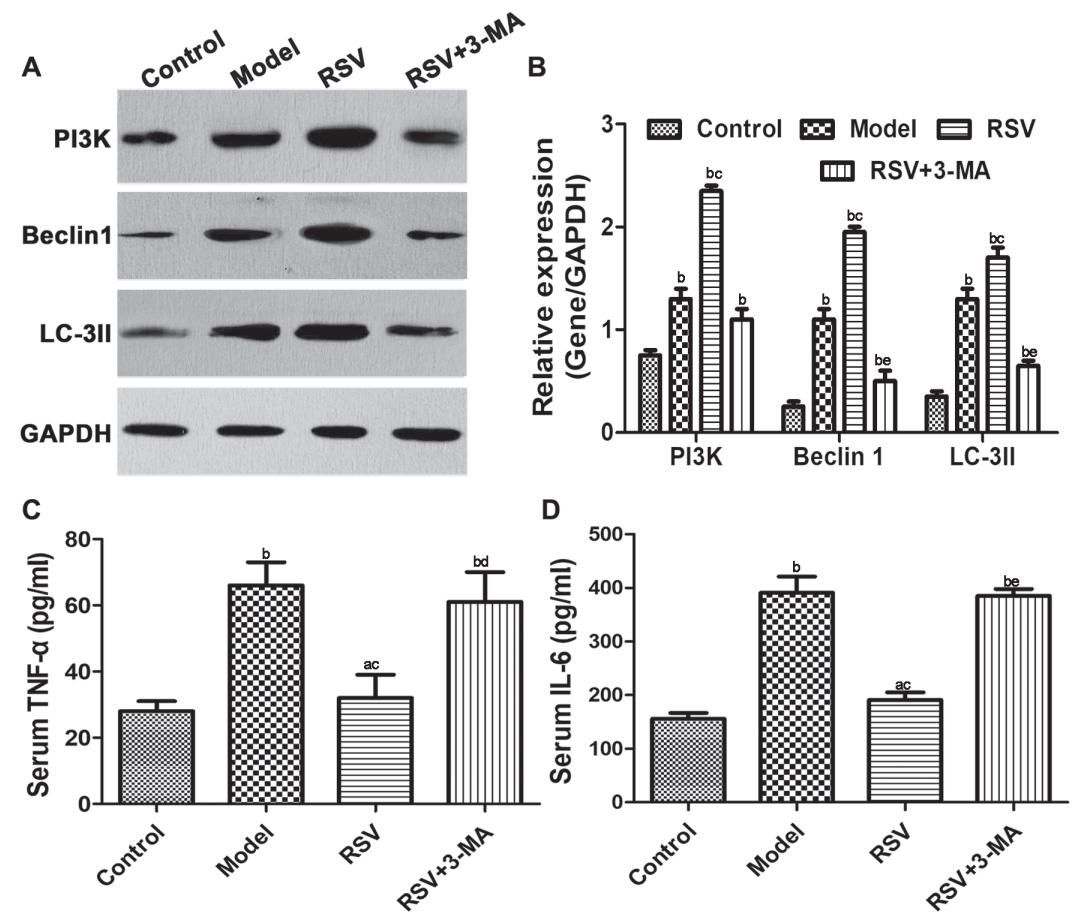

Figure 4. RSV and 3-MA affects the expression of Beclin 1 and LC3-II and the serum levels of TNF- $\alpha$ and IL-6. (A) RSV promoted the expression of PI3K, Beclin 1 and LC-3II, as detected by western blotting. Diabetic rats in the RSV group were given RSV (10 mg/kg/d). Rats in the 3-MA (RSV + 3-MA) group were administered 3-MA $(0.015 \mathrm{~g} / \mathrm{kg} / \mathrm{d})$, based on the RSV $(10 \mathrm{mg} / \mathrm{kg} / \mathrm{d})$ injection. The Control and Model groups were injected with the same amount of physiological saline. After 7 days, LAD coronary artery was performed on rats in the Model, RSV and 3-MA groups to stimulate myocardial I/R injury. The control group underwent a sham surgery. Following I/R for $2 \mathrm{~h}$, proteins were extracted for western blotting. (B) The relative expression of targets was normalized to GAPDH. RSV inhibited serum (C) TNF- $\alpha$ and (D) IL-6, as detected by ELISA. ${ }^{a} \mathrm{P}<0.05$ and ${ }^{\mathrm{b}} \mathrm{P}<0.01$ vs. the control group; ${ }^{\mathrm{c}} \mathrm{P}<0.01$ vs. the Model group; ${ }^{\mathrm{d}} \mathrm{P}<0.05$ and ${ }^{\mathrm{e}} \mathrm{P}<0.01$ vs. the RSV group. RSV, resveratrol; 3-MA, 3-methyladenine; LC3-II, light chain 3-II; LAD, left anterior descending; $\mathrm{I} / \mathrm{R}$, ischemia/reperfusion; TNF, tumor necrosis factor; IL, interleukin; PI3K, phosphatidylinositol 3 kinase.

increased, as compared with that in the RSV group sections $(\mathrm{P}<0.05)$. Fig. 3B shows the infarct size rate $(\%)$. These data indicated that RSV mitigated the myocardial infarct size caused by ischemia and $\mathrm{I} / \mathrm{R}$, but 3-MA suppressed the role of RSV.
$R S V$ protects against I/R injury by inducing autophagy and blocking cytokines. A previous study revealed the relationship between ischemia and I/R and autophagy (20). To explore the mechanism by which RSV mitigates ischemia and I/R, the expression levels of autophagy markers Beclin 1 and LC-3II 
were mainly detected. The protein levels of PI3K, Beclin 1 and LC-3II were significantly upregulated in the Model group compared with the Control ( $\mathrm{P}<0.01$; Fig. 4A and B). Furthermore, the addition of RSV further enhanced those levels. However, treatment with the autophagy inhibitor 3-MA significantly blocked the upregulation induced by RSV $(\mathrm{P}<0.01)$. The role of RSV on cytokines was further investigated. The serum level of TNF- $\alpha$ in the Model group was significantly increased compared with the control group $(\mathrm{P}<0.01$; Fig. 4C). However, the addition of RSV blocked the increase of TNF- $\alpha$ and 3-MA application inhibited the function of RSV. A similar trend was observed in serum IL-6 levels (Fig. 4D). These data suggest that RSV promoted the expression of Beclin 1 and LC-3II but blocked the serum levels of TNF- $\alpha$ and IL- 6 .

\section{Discussion}

Cardiovascular disease, including I/R injury, has been associated with high mortality worldwide. Microvascular complications are considered to play a major role in diabetic complications (21) and diabetes has been reported to cause an enhanced vulnerability of the myocardium to myocardial I/R injury (22). The aim of the present study was to explore the function of RSV against myocardial I/R injury in diabetes and to reveal the underlying mechanisms. During the heart hemodynamic function examination, the levels of LVSP, $+\mathrm{dP} / \mathrm{dt}$ and $-\mathrm{dP} / \mathrm{dt}$ were blocked by LAD and reperfusion. The RSV injection mitigated that blockage. However, the addition of 3-MA suppressed the function of RSV. In addition, the I/R injury-induced increase in infarct size was blocked by RSV and 3-MA reversed the function of RSV. These data provided new evidence on the function of RSV on myocardial I/R injury in diabetes. Furthermore, it was found that I/R injury enhanced the expression of Beclin 1 and LC3II and serum levels of TNF- $\alpha$ and IL-6. The application of RSV promoted the elevation of Beclin 1 and LC-3II but decreased the serum levels of TNF- $\alpha$ and IL-6. However, 3-MA reversed the effect of RSV. These results showed that RSV ameliorates diabetes-induced I/R injury through inducing autophagy and blocking cytokines TNF- $\alpha$ and IL-6.

Dietary phytochemicals are becoming popular as functional foods and nutritional supplements. RSV is a naturally occurring polyphenol (23), now considered a potent cardioprotective compound (24). A previous study showed that the beneficial effects of RSV on myocardial I/R injury might have a multifactorial basis (25). Certain reports have revealed that RSV is involved in cardioprotection via regulating the expression of thioredoxin-1 (26), hemeoxygenase-1 (27) and vascular endothelial growth factor (28), among others. Despite significant advances in the understanding of the function and mechanism of RSV against myocardial I/R injury, the effects and molecular basis of RSV on myocardial I/R injury under diabetic conditions remain unclear. In the present study, it was found that the application of RSV clearly elevated the levels of LVSP, $+\mathrm{dP} / \mathrm{dt}$ and $-\mathrm{dP} / \mathrm{dt}$, and ameliorated the infarct size in diabetic myocardial I/R injury. These results indicated that RSV plays a role in the mechanisms of resistance to I/R injury in diabetic rats, which was consistent with a previous report (29).
Autophagy has been reported to be activated following myocardial I/R injury in cardiomyocytes $(30,31)$. The regulation of autophagy provided a new strategy for the treatment of myocardial ischemia and heart failure (32). The blockage of autophagy is commonly achieved through the inhibition of class III PI3K via a pharmacological agent 3-MA (33). In this study, the administration of 3-MA improved the infarct size, as compared with the RSV-treated group, suggesting the close association between RSV and autophagy. A previous study showed that RSV attenuates cardiac dysfunction associated with the regulation of autophagic flux in diabetic mice (17), which was consistent with the results of the present study. Beclin-1 is the mammalian homologue of the yeast Atg6 (34) and the activation of Beclin-1 at the site of injury denotes the induction of autophagy (35). Similar to Beclin-1, LC3-II, a mammalian homologue of yeast Atg8p, is also a credible marker for autophagy (30). It was found herein that the application of RSV significantly promoted the expression of Beclin 1 and LC3-II, and that the inhibition of autophagy achieved by 3-MA significantly blocked the RSV-induced upregulation of Beclin 1 and LC3-II. These findings were consistent with those of a previous study showing that RSV induced autophagy, as evidenced by the abilities to activate autophagic markers LC3-II and Beclin-1 (36,37). It has been reported that RSV attenuated I/R-induced NLRP3 inflammasome-derived inflammation through SIRT1-dependent autophagy activity (13). A number of studies have shown that the upregulation of TNF- $\alpha$ and IL- 6 directly leads to organ damage by aggravating the inflammatory response (38-40). In this study, it was found that RSV mitigated the I/R-induced release of TNF- $\alpha$ and IL-6, which was in accordance with the above study. In combination, these findings provided new evidence that RSV induced autophagy and modulated inflammatory response in myocardial I/R injury in diabetes.

The successful application of RSV lies in understanding its mechanisms of action through direct and indirect interactions with pathways. In the present study, RSV decreased the levels of LVSP, $+\mathrm{dP} / \mathrm{dt}$ and $-\mathrm{dP} / \mathrm{dt}$, as well the I/R injury-induced infarct size of the diabetic myocardium. The effects of RSV on I/R injury were reversed by 3-MA. RSV induced the activation of autophagy via the upregulation of Beclin 1 and LC3-II and inhibited inflammatory response via the blockage of TNF- $\alpha$ and IL-6. In conclusion, RSV was found to alleviate I/R injury of the diabetic myocardium via inducing autophagy and blocking inflammatory response.

\section{Acknowledgements}

Not applicable.

\section{Funding}

The present study was supported by the Foundation of science and Technology bureau of Taizhou (grant no. 162yw04).

\section{Availability of data and materials}

All data generated or analyzed during this study are included in this published article. 


\section{Authors' contributions}

XQ constructed the animal model of myocardial I/R, analyzed the data and wrote the original manuscript; XC and QS performed the measurement of hemodynamic function of the heart and myocardial infarct size; XW and DW carried out the ELISA assays and western blot analyzes. LY designed the study and corrected the manuscript. All authors read and approved the final version of the manuscript.

\section{Ethics approval and consent to participate}

All experimental procedures were approved by the Experimental Animal Ethics Committee of Municipal Hospital of Taizhou (Zhejiang, China).

\section{Patient consent for publication}

Not applicable.

\section{Competing interests}

The authors declare that they have no competing interests.

\section{References}

1. Franz MJ, Boucher JL, Rutten-Ramos S and VanWormer JJ: Lifestyle weight-loss intervention outcomes in overweight and obese adults with type 2 diabetes: A systematic review and meta-analysis of randomized clinical trials. J Acad Nutr Diet 115: 1447-1463, 2015.

2. de Almeida Barreto FK, Montenegro RM Jr, Fernandes VO, Oliveira R, de AraUjo Batista LA, Hussain A and de Góes Cavalcanti LP: Chikungunya and diabetes, what do we know? Diabetol Metab Syndr 10: 32, 2018.

3. Thirunavukkarasu M, Penumathsa SV, Koneru S, Juhasz B, Zhan L, Otani H, Bagchi D, Das DK and Maulik N: Resveratrol alleviates cardiac dysfunction in streptozotocin-induced diabetes: Role of nitric oxide, thioredoxin, and heme oxygenase. Free Radic Biol Med 43: 720-729, 2007.

4. Sotníková R, Nedelčevová J, Navarová J, Nosálová V, Drábiková K, Szöcs K, Křenek P, Kyseĺlová Z, Bezek S, Knezl V, et al: Protection of the vascular endothelium in experimental situations. Interdiscip Toxicol 4: 20-26, 2011.

5. Bulhak AA, Jung C, Ostenson CG, Lundberg JO, Sjoquist PO and Pernow J: PPAR-alpha activation protects the type 2 diabetic myocardium against ischemia-reperfusion injury: Involvement of the PI3-Kinase/Akt and NO pathway. Am J Physiol Heart Circ Physiol 296: H719-H727, 2009.

6. Wang $\mathrm{J}$ and Li J: Activated protein C: A potential cardioprotective factor against ischemic injury during ischemia/reperfusion. Am J Transl Res 1: 381-392, 2009.

7. Korkmaz-Icöz S, Lehner A, Li S, Vater A, Radovits T, Hegedús P, Ruppert M, Brlecic P, Zorn M, Karck M and Szabó G: Mild type 2 diabetes mellitus reduces the susceptibility of the heart to ischemia/reperfusion injury: Identification of underlying gene expression Changes. J Diabetes Res 2015: 396414, 2015.

8. Wang S, Wang C, Yan F, Wang T, He Y, Li H, Xia Z and Zhang Z: $\mathrm{N}$-acetylcysteine attenuates diabetic myocardial ischemia reperfusion injury through inhibiting excessive autophagy. Mediators Inflamm 2017: 9257291, 2017.

9. Moens AL, Claeys MJ, Timmermans JP and Vrints CJ: Myocardial ischemia/reperfusion-injury, a clinical view on a complex pathophysiological process. Int J Cardiol 100: 179-190, 2015.

10. Feyzizadeh S and Badalzadeh R: Application of ischemic postconditioning's algorithms in tissues protection: Response to methodological gaps in preclinical and clinical studies. J Cell Mol Med 21: 2257-2267, 2017.

11. Sabe AA, Elmadhun NY, Dalal RS, Robich MP and Sellke FW: Resveratrol regulates autophagy signaling in chronically ischemic myocardium. J Thorac Cardiovasc Surg 147: 792-799, 2014.
12. Fernandes GFS, Silva GDB, Pavan AR, Chiba DE, Chin CM and Dos Santos JL: Epigenetic regulatory mechanisms induced by resveratrol. Nutrients 9: pii: E1201, 2017.

13. He Q, Li Z, Wang Y, Hou Y, Li L and Zhao J: Resveratrol alleviates cerebral ischemia/reperfusion injury in rats by inhibiting NLRP3 inflammasome activation through Sirt1-dependent autophagy induction. Int Immunopharmacol 50: 208-215, 2017.

14. Mizushima N: A brief history of autophagy from cell biology to physiology and disease. Nat Cell Biol 20: 521-527, 2018.

15. Gottlieb RA, Finley KD and Mentzer RM Jr: Cardioprotection requires taking out the trash. Basic Res Cardiol 104: 169-180, 2009.

16. Wang R, Liu YY, Liu XY, Jia SW, Zhao J, Cui D and Wang L: Resveratrol protects neurons and the myocardium by reducing oxidative stress and ameliorating mitochondria damage in a cerebral ischemia rat model. Cell Physiol Biochem 34: 854-864, 2014.

17. Wang B, Yang Q, Sun YY, Xing YF, Wang YB, Lu XT, Bai WW, Liu XQ and Zhao YX: Resveratrol-enhanced autophagic flux ameliorates myocardial oxidative stress injury in diabetic mice. J Cell Mol Med 18: 1599-1611, 2014.

18. Mukhopadhyay P, Pacher P and Das DK: MicroRNA signatures of resveratrol in the ischemic heart. Ann N Y Acad Sci 1215: 109-116, 2011.

19. Zhang S, Yang J, Li H, Li Y, Liu Y, Zhang D, Zhang F, Zhou W and Chen X: Skimmin, a coumarin, suppresses the streptozotocin-induced diabetic nephropathy in wistar rats. Eur J Pharmacol 692: 78-83, 2012.

20. Wu H, Ye M, Liu D, Yang J, Ding JW, Zhang J, Wang XA, Dong WS and Fan ZX: UCP2 protect the heart from myocardial ischemia/reperfusion injury via induction of mitochondrial autophagy. J Cell Biochem, May 13, 2019 (Epub ahead of print).

21. Joshi MS, Williams D, Horlock D, Samarasinghe T, Andrews KL, Jefferis AM, Berger PJ, Chin-Dusting JP and Kaye DM: Role of mitochondrial dysfunction in hyperglycaemia-induced coronary microvascular dysfunction: Protective role of resveratrol. Diab Vasc Dis Res 12: 208-216, 2015.

22. Donahoe SM, Stewart GC, McCabe CH, Mohanavelu S, Murphy SA, Cannon CP and Antman EM: Diabetes and mortality following acute coronary syndromes. JAMA 298: 765-775, 2007.

23. Sabe AA, Elmadhun NY, Robich MP, Dalal RS and Sellke FW: Does resveratrol improve insulin signaling in chronically ischemic myocardium? J Surg Res 183: 531-536, 2013.

24. Cho S, Namkoong K, Shin M, Park J, Yang E, Ihm J, Thu VT, Kim HK and Han J: Cardiovascular protective effects and clinical applications of resveratrol. J Med Food 20: 323-334, 2017.

25. Penumathsa SV and Maulik N: Resveratrol: A promising agent in promoting cardioprotection against coronary heart disease. Can J Physiol Pharmacol 87: 275-286, 2009.

26. Welsh SJ, Bellamy WT, Briehl MM and Powis G: The redox protein thioredoxin-1 $(\operatorname{Tr} x-1)$ increases hypoxia-inducible factor 1alpha protein expression: Trx-1 overexpression results in increased vascular endothelial growth factor production and enhanced tumor angiogenesis. Cancer Res 62: 5089-5095, 2002.

27. Penumathsa SV, Koneru S, Samuel SM, Maulik G, Bagchi D, Yet SF, Menon VP and Maulik N: Strategic targets to induce neovascularization by resveratrol in hypercholesterolemic rat myocardium: Role of caveolin-1, endothelial nitric oxide synthase, hemeoxygenase-1, and vascular endothelial growth factor. Free Radic Biol Med 45: 1027-1034, 2008.

28. Sakamoto H, Nakamura T, Akuzawa N, Masuda H, Sumino H, Saito Y, Ohyama Y, Kurashina T, Tamura J and Kurabayashi M: Reciprocal expression of vascular endothelial growth factor and nitric oxide synthase by coronary arterial wall cells during chronic inhibition of nitric oxide synthesis in rats. Nephron 92: 472-474, 2002.

29. Yang Q, Wang HC, Liu Y, Gao C, Sun L and Tao L: Resveratrol cardioprotection against myocardial ischemia/reperfusion injury involves upregulation of adiponectin levels and multimerization in type 2 diabetic mice. J Cardiovasc Pharmacol 68: 304-312, 2016.

30. Martinet W, Knaapen MW, Kockx MM and De Meyer GR: Autophagy in cardiovascular disease. Trends Mol Med 13: 482-491, 2007.

31. Gustafsson AB and Gottlieb RA: Autophagy in ischemic heart disease. Circ Res 104: 150-158, 2009.

32. Nishida K, Yamaguchi O and Otsu K: Crosstalk between autophagy and apoptosis in heart disease. Circ Res 103: 343-351, 2008. 
33. Lekli I, Ray D, Mukherjee S, Gurusamy N, Ahsan MK, Juhasz B, Bak I, Tosaki A, Gherghiceanu M, Popescu LM and Das DK: Co-ordinated autophagy with resveratrol and $\gamma$-tocotrienol confers synergetic cardioprotection. J Cell Mol Med 14: 2506-2518, 2010

34. Zalckvar E, Berissi H, Eisenstein $M$ and Kimchi A: Phosphorylation of Beclin 1 by DAP-kinase promotes autophagy by weakening its interactions with Bcl-2 and Bcl-XL. Autophagy 5: 720-722, 2009.

35. Matsui Y, Takagi H, Qu X, Abdellatif M, Sakoda H, Asano T, Levine B and Sadoshima J: Distinct roles of autophagy in the heart during ischemia and reperfusion: Roles of AMP-activated protein kinase and Beclin 1 in mediating autophagy. Circ Res 100: 914-922, 2007.

36. Xuan W, Wu B, Chen C, Chen B, Zhang W, Xu D, Bin J and Liao Y: Resveratrol improves myocardial ischemia and ischemic heart failure in mice by antagonizing the detrimental effects of fractalkine*. Crit Care Med 40: 3026-3033, 2012.
37. Chen-Scarabelli C, Agrawal PR, Saravolatz L, Abuniat C, Scarabelli G, Stephanou A, Loomba L, Narula J, Scarabelli TM and Knight R: The role and modulation of autophagy in experimental models of myocardial ischemia-reperfusion injury. J Geriatr Cardiol 11: 338-348, 2014.

38. Liu D, Jin X, Zhang C and Shang Y: Sevoflurane relieves hepatic ischemia-reperfusion injury by inhibiting the expression of Grp78. Biosci Rep 38: pii: BSR20180549, 2018.

39. Liu L, Li X, Yang J, Chai J, Yu Y, Duan H, Song H, Feng R, Wang $\mathrm{T}$, Yin $\mathrm{H}$, et al: Comparison of systemic inflammation response and vital organ damage induced by severe burns in different area. Int J Clin Exp Pathol 8: 6367-6376, 2015.

40. Zhang S, Xin H, Li Y, Zhang D, Shi J, Yang J and Chen X: Skimmin, a coumarin from hydrangea paniculata, slows down the progression of membranous glomerulonephritis by anti-inflammatory effects and inhibiting immune complex deposition. Evid Based Complement Alternat Med 2013: 819296, 2013. 